\title{
China en los intersticios de la crisis del multilateralismo y la globalización neoliberal: La Franja y la Ruta en Europa y el caso italiano
}

\section{China in the Interstices of the Crisis of Multilateralism and Neoliberal Globalization: The Belt and Road in Europe and the Italian Case}

\author{
Javier Vadell ${ }^{1}$ \\ Pontificia Universidad Católica de Minas Gerais (Brasil) \\ Maria Francesca Staiano ${ }^{2}$ \\ Universidad Nacional de La Plata (Argentina)
}

Recibido: 22-09-20

Aceptado: 18-10-20

\begin{abstract}
${ }^{1}$ (javier.vadell@gmail.com). Profesor Asociado del Departamento de Relaciones Internacionales de la Pontificia Universidad Católica de Minas Gerais. Destacan entre sus publicaciones las siguientes: Vadell, Javier; Giaccaglia, Clarisa: El rol de Brasil en el regionalismo latinoamericano: la apuesta por una inserción internacional solitaria y unilateral. FORO INTERNACIONAL, v. 60, pp. 1041-1080, 2020. Doi: 10.24201/fi.v60i3.2770; Vadell, Javier; LoBrutto, G.; Leite, A.: The Chinese South-South Development Cooperation: An Assessment of the Structural Transformation. Revista Brasileira de Politica Internacional, v. 63, pp. 1-22, 2020. Doi: 10.1590/0034-7329202000201; Vadell, Javier: La iniciativa BRICS y China: entre la emergencia y la irrelevancia. Nova Economia (UFMG), v. 29, pp. 401-428, 2019. Doi: https://doi.org/10.1590/0103-6351/5410; Vadell, Javier: 2019: China in Latin America: South-South Cooperation with Chinese Characteristics, Latin American Perspectives 46 (2):107-125. Doi: Vadell, https://doi.org10.1177/0094582X18815511.

ORCID: https://orcid.org/0000-0002-5398-6083.

2 (mf.staiano@gmail.com). Coordinadora del Centro de Estudios Chinos, Instituto de Relaciones Internacionales, Universidad Nacional de La Plata (IRI-UNLP). Algunos de sus principales trabajos son: Staiano, Maria Francesca y Bogardo Bordazar, Laura L.: La iniciativa la Franja y la Ruta: innovación propulsora de los procesos de integración regional a nivel global. Los casos de Europa y América Latina. 一带一路倡议: 将区域一体化升级至全球层面 的推动性创新。欧洲与拉美的案例, en Staiano, Maria Francesca - Bogado Bordazar, Laura L. - Caubet, Matías: "China: una nueva estrategia geopolítica global (la iniciativa la Franja y la Ruta)", Universidad Nacional de La Plata Press, Mayo 2019, pp. 135-145; Staiano, Maria Francesca y Marcelli, Fabio, “COVID-19全球疫情的挑战、反思与展望一以意大利为 视角的观察” (Challenges, Reflections and Prospects of the COVID-19 Global Pandemic. An Observation from Italy's Perspective), en Chinese Review of International Law (国际法研究编辑部 国际法研究), Vol. 4, July 2020, disponible en https://mp.weixin.qq.com/s/OWRdPeJ1SFaRlaR8S_50gQ; Staiano, Maria Francesca: La relaciones internacionales entre China y América Latina: encontrando un camino común hacia un nuevo orden mundial, en Humania del Sur - Revista de Estudios Latinoamericanos, Africanos y Asiáticos, n. 25, Julio-Diciembre 2018, Universidad de Los Andes, Mérida, Venezuela.
\end{abstract}

ORCID: https://orcid.org/0000-0002-2015-319X.. 


\title{
Resumen
}

El artículo tiene como objetivo analizar la presencia de China en Europa a través de la Iniciativa de la Franja y la Ruta (IFR) en un contexto de crisis de la globalización neoliberal y del multilateralismo. Las preguntas iniciales son ¿de qué manera la IFR está contribuyendo a reconfigurar la globalización y el regionalismo en la Unión Europea (UE) y cuáles son los impactos en la UE de este tipo de acuerdos? El artículo enfoca en el caso de Italia como análisis descriptivo y exploratorio, que si bien no es el único país de la UE que firmó un Memorándum de Entendimiento de la IFR, fue el único del G7 que lo hizo. Finalmente, el artículo destaca cómo la pandemia del COVID19 tornó más compleja la relación China-Europa, provocando reacciones diferenciadas entre la UE e Italia

Palabras-clave: China, Europa, Franja y La Ruta, Italia, Globalización.

\begin{abstract}
The article aims to analyze the presence of China in Europe through the Belt and Road Initiative (BRI) in a landscape of crisis of neoliberal globalization and multilateralism. The initial questions are in what way the BRI is contributing to reconfigure the globalization and regionalism in the European Union (EU)? and what are the effects on the EU of this type of agreements? The article focuses on Italian case study as a descriptive and exploratory analysis. Italy, although it is not the only EU country that signed the BRI Memorandum of Understanding, it was the only G7 country to signed it. Finally, the article highlights how the COVID19 pandemic made the China-Europe relationship more complex, causing diverse reactions between the EU and Italy
\end{abstract}

Key-words: China, Europe, Belt and Road, Italy, Globalization.

\section{Introducción}

Las relaciones internacionales contemporáneas de la República Popular de China (RPC) con Europa atraviesan un período de compleja relación en consonancia con las transformaciones de la economía global en épocas de pandemia. La expansión del gran proyecto euroasiático chino, la Iniciativa de la Franja y la Ruta (IFR) ${ }^{3}$ o nuevas Rutas de la Seda, han añadido un ingrediente adicional a la complejidad de la relación China-Europa.

Entre los estudiosos y analistas de relaciones internacionales existe un supuesto básico que entiende la expansión de China, en las esferas económica y

\footnotetext{
${ }^{3}$ Belt and Road Initiative (BRI por su sigla en inglés).
} 
política global, como una de las variables más relevantes en las transformaciones del orden global internacional y de las instituciones de gobernanza global. Estos cambios tienen implicaciones importantes en términos de difusión de ideas y prácticas, conformación de identidades y valores y cumplimiento de reglas y normas entre actores internacionales en la medida que China se consolida no ya como un rule shaper, sino como un rule maker.

El término globalización es uno de los más controvertidos, ambiguos y polisémicos del discurso político contemporáneo. Las relaciones internacionales y la economía política internacional han definido de manera más sistemática este concepto solo a finales de la década de 1990, en un momento crucial del proceso de expansión del capitalismo a escala global. Un proceso de "expansión financiera sistémica" como definido por Arrighi, Silver y Ahmad (1999, p.31), que aquí denominamos financiarización. Son momentos de transformación estructural del SMM que antecede al "caos sistémico" (Arrighi, Silver y Ahmad 1999, p.32), que se manifiesta en la interacción entre una mayor intensidad de la competición interestatal e interempresarial.

Cuando afirmamos que el proyecto chino de la IFR está reconfigurando la geografía económica y la geopolítica global es menester hacer hincapié en los componentes 'espacio' y 'tiempo' de este proceso en curso. De esta manera, nuestro punto de partida son las controversias presentadas por autores de la economía política crítica como Arrighi, Silver y Ahmad (1999) que a finales de la década de 1990 anticipaban el rol prominente de China en el siglo XXI frente a los cambios en el equilibrio de poder entre los Estados. Nuestra premisa es que la globalización escondió el poder de los Estados nacionales y que atravesamos una fase de transición del ciclo de dominación Occidental en el Sistema Mundial Moderno (SMM). Esta premisa es el telón de fondo de nuestras principales indagaciones.

Este punto de partida nos lleva a preguntas que no tienen respuestas fáciles en un contexto de crisis de la globalización neoliberal y del multilateralismo. ¿De qué manera la presencia de China en Europa a través de la IFR está contribuyendo a reconfigurar la globalización y el regionalismo en la UE? ¿Cuáles son los impactos en la UE de los acuerdos que algunos de sus miembros firmaron con China, específicamente en el caso de Italia? Y, finalmente, ¿qué nos puede enseñar el caso italiano, que si bien no es el único país de la UE que firmó un Memorándum de Entendimiento $\left(\mathrm{MoU}^{4}\right)$ de la IFR, es el único del G7 que lo hizo, a pesar de las advertencias de los EEUU? La pandemia, ¿modificó o exhibió de manera más cruda la complejidad de la relación China-Europa? ¿De qué manera?

La IFR está reconfigurando la geografía económica por medio de masivas inversiones en infraestructura que no tienen precedente en la actual fase del

\footnotetext{
${ }^{4}$ Dejaremos la sigla en inglés, Memorandum of Understanding (MoU).
} 
capitalismo global y Europa es el punto geográfico crucial de conexión con China de un proyecto que comenzara como euroasiático. Nuestra primera hipótesis general es que la IFR representa, una respuesta a la globalización neoliberal (desembedded liberalism) fundamentada en una propuesta de interconexión con principios liberales integrados (embedded liberalism), que surgió como iniciativa regional (Eurasia) y se expandió globalmente. Por otro lado, también es un desafío para el regionalismo europeo. La UE está lidiando con el complejo relacionamiento con China, que se presenta económicamente relevante y políticamente arriesgado. La pandemia del COVID-19 expuso aún más algunas paradojas que ilustramos con el caso italiano.

En el caso europeo en particular, la IFR ha generado una dinámica que está sacudiendo a la UE de dos maneras: por un lado, pone en tela de juicio la brecha económica entre Europa del Este y Europa Occidental, evidenciando una "intromisión" de China que busca darle visibilidad y relevancia a esta "zona gris" de Europa, excluida política y económicamente de la UE, pero indirectamente sometida a esta (Staiano \& Bogado Bordazar, 2019); por otro lado, dentro de la EU se ha manifestado una dicotomía cada vez más clara entre países del Norte, con la alianza Alemania-Francia como eje central decisional, y los países del Sur (Italia, España, Grecia y Portugal), que parece replicar una doble dimensión de la integración europea, ya definida por muchos autores como dinámica de "distintas velocidades", caracterizada por una relación centro/periferia.

El plan estratégico UE-China ${ }^{5}$ debería ser el marco normativo más general. Sin embargo, la firma del MoU de la IFR por parte de Italia tuvo repercusiones y supuso un gran desafío para la UE en dos planos: por un lado, se supone y se está negociando una acción europea conjunta para relacionarse con China, para evitar las relaciones bilaterales China-país; por otro lado, la estrategia de la UE no considera de la misma manera a sus miembros.

Para este propósito, este artículo, en la siguiente sección, definirá algunos conceptos operacionales que nos auxiliarán para comprender el fenómeno de la expansión económica de China a escala global y regional, auxiliados por el marco de la teoría crítica del SMM y por la categorización de conceptos como "globalización" y "liberalismo integrado". La sección tercera define el liberalismo integrado con características chinas vinculado al proyecto IFR. La cuarta sección enfoca y analiza el impacto de la llegada de la IFR a Europa, a la UE y las particularidades del caso italiano. Dejaremos la parte final para algunas conclusiones.

\footnotetext{
${ }^{5}$ EU-China 2020 Strategic Agenda for Cooperation, disponible en http://eeas.europa.eu/archives/ docs/china/docs/eu-china_2020_strategic_agenda_en.pdf

${ }^{6}$ Embedded liberalism en inglés, derivado del concepto de Karl Polanyi.
} 


\section{2. ¿Globalización neoliberal versus globalización China?}

Giovanni Arrighi (1996, p. 174) afirmó que, en los ciclos hegemónicos del capitalismo, las fases productivas y las fases de financiarización se repitieron en la historia como un fenómeno recurrente. Es decir, los ciclos de acumulación y hegemonía en el capitalismo coinciden con procesos de fases de expansión productiva y de decadencia que se inician en procesos de financiarización. Por su parte, Samir Amin expresa: "resta saber si la recurrencia es una regularidad - de forma cíclica- y si es fecundo destacar esto, borrando las especificidades de la financiarización (de la globalización neoliberal contemporánea) en las diferentes fases del desarrollo capitalista" (Amin, 2006, pp. 142-143). La especificidad de la expansión financiera contemporánea atraviesa una fase crítica donde los intersticios de la historia del capitalismo preanuncian una señal de crisis hegemónica (Arrighi, 2007; Arrighi, Silver, \& Ahmad, 1999, p. 272). Hace más de veinte años Arrighi llamaba la atención para un escenario en donde el crecimiento del Este de Asia, que él denominaba la "Gran China" (Hong Kong, Taiwán y la RPC), sería crucial para entender el desgaste y la crisis de la globalización neoliberal.

De esta manera y acorde a aquel escenario planteado, la proyección global del IFR tiene características diferentes de la globalización neoliberal y se desarrolla en los intersticios del SMM. La IFR se presenta también es una "nueva" globalización "abierta e integradora" (Weidong Liu \& Michael Dunford, 2016), basada en la interconectividad e inversiones en infraestructura, pero con el control estatal de las finanzas. Sus principales rasgos son: 1) una nueva configuración espacial con la abertura de nuevas rutas de transporte marítimos y terrestres (corredores) que comienza como proyecto euroasiático y luego se expande globalmente, cuando América Latina y el Caribe (ALC) son invitados en 2017 (Vadell, 2018); 2) transformaciones institucionales de la gobernanza económica global, vinculadas a la cooperación financiera-bancaria con control estatal.

\subsection{Rastreando la globalización neoliberal}

David Held y Anthony McGrew (2000) realizan un interesante esfuerzo en sistematizar y analizar el concepto polisémico de globalización, promovieron un rico debate. Para estos autores, la metamorfosis del significado de globalización implica inherentemente una transformación. Siendo más precisos, la globalización significa una "ampliación, profundización y aceleración de la interconectividad del mundo en todos los aspectos de la vida social contemporánea, desde lo cultural a lo criminal, desde lo financiero a lo espiritual" (Held \& McGrew, 2000, p. 2). 
Desde la crisis financiera de 2008, la globalización económica está en una encrucijada política y económica (Sheppard, 2016), que se agudizó aún más en el año de la pandemia, 2020. La literatura de economía política internacional (EPI) crítica reconoce que los ciclos expansión de capitalismo pueden caracterizarse como procesos de globalización, es decir, una mayor interdependencia de actores estatales y no estatales producto de una acelerada expansión e intensificación de la acumulación capitalista.

En este sentido, el marco conceptual dialéctico de Karl Polanyi (2000) y su tesis del doble movimiento, como respuestas y oposición a los mercados autorregulados globales, es útil para comprender el movimiento globalizante y los contra-movimientos en escala global, regional y local. El movimiento es caracterizado por una expansión - ampliación geográfica de las relacionas capitalistas- e intensificación -profundización de las relaciones mercantiles (commodification) en las esferas de la vida humana-. Este proceso no es lineal, ni carente de contradicciones. El aporte de Polanyi a nuestra interpretación ayuda a entender la quiebra de la linealidad histórica y metodológica, muy acorde al pensamiento liberal.

Karl Polanyi nos ofrece un escenario magistral de la evolución de lo que él denomina utopía liberal (Polanyi, 2000, p. 18) de finales del siglo XIX a la catástrofe de la década de 1930 del siglo XX, "ligando las dimensiones nacionales del funcionamiento destructivo de los mercados de trabajo, de la tierra y de la moneda a su dimensión mundial" (Amin, 2006, p. 130). El contra-movimiento no ocurre de manera lineal sin conflictos y sin contradicciones.

La globalización se refiere a un proceso creciente de interconectividad "transplanetaria" que afecta a todas las esferas de la vida "en términos de alteración de la espacialidad social, transformando el Estado nacional y el capitalismo" (Scholte, 2005). La IFR se encuadra en este concepto, no solo porque propone una creciente interconectividad, sino porque también recupera la cuestión de la territorialidad. Cairo Carou destaca que "los territorios y la territorialidad humana son construcciones sociales y no han tenido siempre la disposición y características actuales" (Cairo Carou, 2018, p. 27). La territorialidad se relaciona con "una conducta humana que intenta influir, afectar o controlar acciones mediante el establecimiento de un control sobre un área geográfica específica: el territorio" (Sack, apud Cairo Carou, 2018, p. 26).

La globalización neoliberal constituye "una manifestación histórica específica de la economía capitalista" (Cahill, 2014, p. X). Dos elementos clave la caracterizan: primero, es un proceso acelerador de expansión e intensificación de las relaciones capitalistas basada en tres pilares esenciales: privatizaciones, liberalización financiera y comercial y re-regulación de las 
economías nacionales. En segundo lugar, se trata de un proyecto de clase (Cahill, 2014; Harvey, 2005), liderado por los sectores de una clase corporativa financiera, una especie haute finance polanyiana fortalecida.

Siguiendo la dialéctica de Polanyi, el "movimiento" neoliberal se inicia a fines de la década de 1970 con la desregulación financiera que comienza en los Estados Unidos de América (EEUU) y la desintegración del sistema Bretton Woods, abriendo el camino a una "expansión financiera sistémica". La importancia en la promoción de las bondades del mercado autorregulado y del rol protagonista del segmento empresarial financiero en ambos períodos fue crucial. Polanyi destaca el rol crucial e inédito de la Haute Finance entre 1870 y 1929 como articulador esencial y "enlace principal entre la organización política y económica del mundo" (Polanyi, 2000, p. 24).

El 'movimiento' neoliberal contemporáneo, a partir de la década de 1980, provoca un cambio desigual en la balanza de fuerzas entre las clases sociales (trabajadores/empresarios) en el interior de los países desarrollados y en los países en desarrollo. El programa de liberalización financiera estimulado por los gobiernos de Ronald Reagan en los EEUU y Margareth Thatcher en Gran Bretaña fueron los catalizadores de procesos cada vez más dinámicos de movilidad geográfica del capital en su forma productiva (especialmente hacia Asia y esencialmente a China) o financiera, centralizados en el polo financiero de Wall Street y en el papel renovado del dólar. De esta manera, la globalización neoliberal estimula este proceso expansivo con algunas características novedosas y específicas que se aceleran con los avances tecnológicos en las comunicaciones y transportes.

La característica inherente de la globalización neoliberal es la supraterritorialidad, "que substancialmente transciende la geografía territorial" (Scholte, 2005) que fortalece las redes de la transnacionalidad de la nueva Haute Finance. La supraterritorialidad significa transcender la geografía por: 1) la "simultaneidad transmundial" (Oke, 2009) donde los fenómenos sociales se extienden a través del planeta al mismo tiempo y en un mismo marco de referencia temporal; y 2) la "instantaneidad", donde "los fenómenos se mueven entre dos puntos del planeta anulando el tiempo" (Scholte, 2005, p. 4). Es decir, es un proceso que comprime el espacio y altera la temporalidad. Ejemplos de este proceso complejo son las comunicaciones vía internet, los patrones y las actitudes de consumo, donde los productos son coordinados por grandes corporaciones monopólicas u oligopólicas transnacionales con estrategias globales. Las finanzas son el sector de la economía que refleja más cabalmente la supraterritorialidad. Es el sector más dinámico/instantáneo y simultáneo del proceso de expansión capitalista. 


\subsection{Interconectividad y regreso al territorio: China y la IFR}

El rol de la "gran China" de Arrighi en la década de 1990 se transfiguró en la RPC del siglo XXI. El desarrollo económico inédito y la actuación cada vez más activa en las instituciones de gobernanza como rule shaper primero, hasta tornarse en rule maker hoy en día, ha facilitado a China su proceso expansivo. Este ascenso desnudó las contradicciones del capitalismo neoliberal frente a un potencial nuevo ciclo hegemónico. La IFR no significa el congelamiento o anulación de la "supraterritorialidad". Esta es absorbida y adaptada en una nueva propuesta de interconectividad geográficamente localizada y basada en inversiones de infraestructura, transporte y energía, ampliación de redes comerciales y finanzas controladas por el Estado al servicio de estas inversiones.

Es en este complejo escenario de post crisis financiera que surge la IFR en 2013, como resultado de un lento y firme ascenso económico de China. Esta iniciativa es anunciada en el inicio del gobierno Xi Jinping y cuestiona implícitamente los fundamentos de la globalización neoliberal exponiendo la crisis del sistema capitalista global. Este contra movimiento global chino desafía al modelo Occidental, sin proponer explícitamente la destrucción de sus bases institucionales. La IFR trae consigo cambios cualitativos, acompañados de una arquitectura institucional financiera y de fórums paralelos, que algunos consideraron una especie de Plan Marshall con características chinas (Vines, 2017).

La iniciativa de la RPC de la "Franja económica de la Ruta de la Seda y la Ruta de la Seda marítima de Siglo XXI" (IFR) se inaugura en 2013 bajo el liderazgo de China. En ese año, el presidente de la RPC, Xi Jinping, en su visita a Asia Central, introdujo por primera vez el término: "Franja económica de la Ruta de la Seda y la Ruta de la Seda marítima de Siglo XXI"” en la Universidad de Nazarbayev (Astana), en Kazajistán. Ese evento fue el puntapié inicial del proyecto de infraestructura e interconectividad por medio de renovadas rutas de comercio y transporte e inversiones en infraestructura en una escala inédita. En un primer momento ligaría China a Europa por transporte terrestre y marítimo, contemplando el continente africano. Posteriormente, se incorporaría ALC.

LA IFR está dividida en dos componentes fundamentales. En primer lugar, la "Franja económica de la ruta de la seda" (Silk Road Economic Belt), que es una serie de rutas terrestres que conectan la RPC con Europa. En segundo lugar, "la ruta marítima de la seda del siglo XXI" (21st Century Maritime Silk Road), que reorganiza las rutas marítimas que interconectan China, el Sudeste Asiático, el Sur de Asia y el océano Î́ndico con África Oriental hasta llegar al mar Mediterráneo.

\footnotetext{
${ }^{7}$ Silk Road Economic Belt y la 21st-Century Maritime Silk Road.
} 
La iniciativa apunta a la construcción de rutas, ferrocarriles, gasoductos y oleoductos, puertos y nuevas rutas aéreas con el objetivo de crear lazos de cooperación comercial y estimular la producción y la interconectividad. Por otro lado, también se pretende estimular la Ruta de la Seda digital (Digital Silk Road) por medio de la cooperación en economía digital, inteligencia artificial, computación en la nube y ciudades inteligentes (ECLAC, 2018) y la Ruta de la Seda Sanitaria (Health Silk Road) (The Lancet, 2017).

El financiamiento de la IFR está siendo realizado por medio de la red multilateral de Bancos liderada por China. Las principales entidades bancarias son: 1) el Fondo de la Ruta de la Seda (Silk Road Fund); 2) el Banco de Desarrollo de China; 3) El Cexim de China (Export-Import Bank of China); 4) El Banco Asiático de Inversiones en Infraestructura - BAII; 5) el Nuevo Banco de Desarrollo de los BRICS (NBD).

En 2015 el Gobierno chino publicó un documento oficial que destaca cinco objetivos básicos de la iniciativa: 1) coordinación de políticas; 2) conectividad de las instalaciones; 3) comercio; 4) integración financiera; y 5) movilidad (Zotelle, 2018). Como fue destacado por el presidente chino Xi Jinping, la IFR está inspirada en la antigua Ruta de la Seda, pero no pretende replicar ese espacio geográfico de comercio, sino ampliarlo para el mundo todo y abierto a todos los países, promoviendo la cooperación y las ganancias mutuas. El documento del gobierno chino "Vision and Actions" de 2015 destaca que la RPC no quiere restablecer la antigua Ruta de la Seda, sino utilizar la metáfora "[d] el espíritu de la Ruta de la Seda", que remite a la antigüedad, como significado cultural para estimular la cooperación internacional (Liu \& Dunford, 2016, p. 4). En palabras oficiales, el espíritu de la Ruta de la Seda por siglos se basó en la "paz, cooperación, apertura, inclusividad, aprendizaje mutuo y beneficio mutuo" (National Development and Reform Commission, 2015).

De esta manera, se pueden identificar dos aspectos interesantes que diferencian el proyecto chino de la globalización neoliberal. En primer lugar, observamos que la integración de China a la globalización fue parcial y controlada por el Estado/Partido. China continuó fuera de la globalización financiera y cuando lo hizo continuó desarrollando su sistema bancario controlado por el Estado y enfocado en el mercado de crédito interno del país. En el proceso de expansión bancaria hacia el exterior permanece el fuerte control estatal de las instituciones financieras que promueven el desarrollo y las inversiones. El capital privado es muy relevante y siempre está ligado a la planificación del Estado. Esta tendencia parece fortalecerse con la idea de 'Frente Unido' público-privado que la pandemia y la guerra tecnológica con los EEUU acentuaron (Gill, 2020).

En segundo lugar, se observa el fuerte énfasis en las inversiones físicas en infraestructura, característica ausente en la globalización neoliberal, donde 
prima el capital financiero. La crisis económica de 2008 y el rol protagonista de China crearon las condiciones del "doble movimiento". Se trata de un proceso de globalización integrada a escala global con características chinas (Chinese embedded globalization), que emerge con sus contradicciones: no abandona los principios liberales, pero no acepta la libertad de los mercados financieros. La idea de reforzar el libre comercio y la globalización es siempre enfatizada en los discursos oficiales chinos. Xi Jinping fue más explícito a medida que se radicalizaba el discurso proteccionista del presidente de los EEUU, Donald Trump (Xi, 2017a, 2017b).

Cuando se menciona el adjetivo integrado (embedded) le estamos otorgando un sentido conceptual y empírico muy claro: el foco infraestructural y físico de las inversiones de la IFR. Esta iniciativa se puede resumir en la fórmula: 5 x 6 . Es decir, 5 rutas y 6 corredores de interconectividad que están recibiendo la mayor parte de las inversiones en infraestructura.

\section{Liberalismo con caracteríticas chinas}

Existe un debate dentro y fuera de China sobre el cambio en la política externa de la RPC. De una política más cauta y de bajo perfil hacia una de mayor asertividad (Qin, 2014; Yan, 2014). La IFR se encuadra en este cambio que puede entenderse como un proceso en curso de características holísticas impulsados por dos principios fundamentales: 1) la noción del "sueño chino" (Li, 2015), con el espíritu de rejuvenecimiento que ello implica, y 2) la idea de "comunidad de destino compartido para la humanidad".

El "sueño chino" tiene implicaciones directas en la política interior china: prosperidad para el país, preocupación con el medio ambiente y felicidad para sus ciudadanos. No obstante, hay un componente universal: "El sueño chino es un sueño de paz, desarrollo, cooperación y mutuos beneficios para todos" $(\mathrm{Li}, 2015)$. Este es el puntapié inicial de una renovada y rejuvenecida política externa china que reivindica e incorpora la tradición reformista de Deng Xiaoping y la actualiza con un protagonismo creciente (Qin, 2014).

La idea de crear una "comunidad de destino compartido" surgió en septiembre de 2011 en el Libro Blanco sobre el Desarrollo pacífico (ChinaSCPRC, 2011), sostenida después en el discurso de Wen Jiabao durante la XIV Summit China-ASEAN y fue retomada por Hu Jintao en el discurso de apertura del XVIII Congreso nacional del Partido Comunista en 2012 (Hu, 2012), en el que se mejoró la expresión con la fórmula "comunidad humana

${ }^{8}$ Edgar Morin fue el primero en profundizar la noción de "comunidad de destino planetaria" teorizada por Otto Bauer (Schicksalgemeinschaft), posteriormente adoptada por el gobierno chino con la expresión “comunidad de destino compartido para la humanidad" 人类 命运 共同体 (Rénlèi mìngyùn gòngtóngtǐ). (Morin, 2001).

Araucaria. Revista Iberoamericana de Filosofia, Politica, Humanidades y Relaciones Internacionales, año $22, \mathrm{n}^{\circ} 45$. Tercer cuatrimestre de 2020. Pp. 433-455. ISSN 1575-6823 e-ISSN 2340-2199 https://dx.doi.org/10.12795/araucaria.2020.i45.18 
con un futuro compartido", perfeccionada posteriormente por Xi Jinping en su famoso discurso de 2015 con motivo del 70 aniversario de las Naciones Unidas: "construyendo una comunidad de destino compartido para el humanidad" (Xi, 2015), que incluye cinco contenidos: asociación política, seguridad, desarrollo económico, intercambios culturales y medio ambiente. Esta perspectiva ha sido retomada en el discurso ante Naciones Unidas en Ginebra en enero 2017 (Xi, 2017b) y ha sido confirmada en octubre del mismo año la idea de la necesidad esencial de "construir una comunidad de futuro compartido" en el Informe del XIX Congreso Nacional del Partido Comunista (Xi, 2017a).

Luego fue incorporado en la Constitución china con la reforma de marzo de 2018 (Staiano, 2018b) como parte del Pensamiento de Xi Jinping sobre el socialismo con características chinas para una nueva era. De esta manera, la "comunidad de destino compartido para la humanidad", 人类命 运共同体 (Rénlèi mìngyùn gòngtóngtǐ), se presenta como un objetivo clave de las relaciones internacionales de China hacia la creación de un nuevo orden internacional, que incluye también las tres dimensiones chinas de "relacionalidad", "autoridad humana" y "simbiosis" (Staiano, 2018a).

La comunidad de destino compartido para la humanidad representa "una nueva solución de gobernanza global propuesta por China", que prevé la creación de "una comunidad global five-in-one", incluyendo "política, seguridad, economía, cultura y ecología" (Xue, 2017: 337). La "comunidad" se refiere al conjunto de los Estados, pequeños y grandes, que coexisten pacíficamente entre ellos a través de valores comunes inspirados por los países líderes en cuanto responsables del orden internacional (autoridad humana), respetándose y ayudándose mutuamente (simbiosis).

Estos conceptos representan elementos de innovación en el marco de las relaciones internacionales, que en la práctica ya tienen resultados empíricos y marcan el comienzo de un nuevo paradigma de las relaciones internacionales a nivel global, el fundamento de lo que denominamos como embeddedness con características chinas. La IFR representa la materialización para la construcción de una Comunidad de Futuro Compartido para la Humanidad y el sueño chino.

La ascensión económica y política de China es el motor de este proceso que cuestiona los fundamentos del neoliberalismo y el rol de los EEUU. Esto tiene un impacto decisivo en la economía global y en sus fundamentos normativos. La relación de China con Europa en un escenario de crisis de multilateralismo y de la globalización neoliberal acelerada por la pandemia es un capítulo abierto de la historia que merece un detallado análisis. 


\section{La IFR en Europa y el caso Italia. La variable COVID como convergencia de la crisis europea}

El objetivo de esta sección es analizar el caso italiano en la actual coyuntura de expansión de la IFR en la región europea y específicamente de la UE. El hecho que consideremos a la IFR una alternativa a la globalización neoliberal no significa que su expansión global estará exenta de conflictos de carácter variado en el tablero de las Relaciones internacionales: a) en el plano de la geopolítica frente a la rivalidad creciente entre Estados Unidos y China; b) en el plano de los regionalismos, colocando un desafío a las autoridades de la UE; c) en la economía global y la estrategia de las grandes corporaciones; $\mathrm{y}$ d) en el cuestionamiento de las desigualdades intra-regionales e intra-clase.

En la última cumbre UE-China, celebrada en junio 2020, se notó un cambio de postura de las instituciones europeas hacia China, en un sentido más "realista", que probablemente coincide con un cambio de la presidencia en la UE, con el nuevo presidente del Consejo Europeo (CE), Charles Michel, la nueva presidenta de la Comisión Europea (ComE), Ursula von der Leyen, acompañados por el nuevo Alto Representante de la Unión para Asuntos Exteriores y Política de Seguridad, Josep Borrell. La $22^{\circ}$ cumbre UE-China no sólo no ha producido un joint statement, sino que los representantes de la UE en la reunión han usado expresiones más duras, en la línea de von der Leyen, definiendo a China como "rival sistémico". La discusión se trabó en términos de la "defensa de los intereses y valores europeos" (EU-China, 2020), donde Michel destacó diferencias entre la UE y China: "Comprometerse y cooperar con China es tanto una oportunidad como una necesidad. Pero, al mismo tiempo, tenemos que reconocer que no compartimos los mismos valores, sistemas políticos o enfoque del multilateralismo. Participaremos de manera lúcida y segura, defendiendo con firmeza los intereses de la UE y manteniéndonos firmes en nuestros valores".

Esta cumbre se ha caracterizado por reclamos de la UE con relación a compromisos que China no habría cumplido. Entre estos, el más relevante son los subsidios estatales del gobierno chino a las empresas y la falta de reciprocidad para que las empresas extranjeras (europeas) tengan acceso al mercado de China. De hecho, los representantes europeos han destacado la necesidad de avanzar en las negociaciones sobre un acuerdo de inversión global UE-China, que garantice la igualdad de condiciones y aborde las asimetrías de acceso al mercado.

Dos años antes, el MoU entre UE y China de 2017 (EC-China, 2017) no tuvo una respuesta satisfactoria en Europa. En marzo de 2019, el Parlamento europeo (PE) y el Consejo de la Unión Europea (CUE) sancionaron el 
Reglamento $452^{9}$, el cual estipula que: "La inversión extranjera directa forma parte de la política comercial común. De conformidad con el artículo 3, apartado 1, e), del TFUE, la Unión tiene competencia exclusiva en lo que respecta a la política comercial común" ${ }^{10}$. De acuerdo con el Reglamento, los controles de seguridad son necesarios en una serie de áreas que incluyen: suministro de alimentos, energía y materias primas; acceso a información sensible como datos personales; infraestructura crítica como transporte, energía, agua, comunicaciones, defensa; tecnologías críticas como inteligencia artificial, robótica, ciberseguridad, aeroespacial, nanotecnología y biotecnología; y la libertad y el pluralismo de los medios de comunicación ${ }^{11}$. Por lo tanto, el Reglamento otorga poderes significativos a los organismos de la UE. Un aspecto interesante que muestra la autonomía de la política externa italiana es que el Reglamento 452 es del 19 de marzo en marzo de 2019 y la visita del presidente de China Xi Jinping a Italia, que implicó la firma del Memorándum de Entendimiento (MoU) de la IFR, fue el 23 de marzo.

La adhesión de Italia a la IFR ha sido muy debatida entre las distintas facciones del Parlamento italiano y, sobre todo, en el marco de la UE. Muchos países miembros se han expresado en contra de la firma de Italia porque eso manifestaría una acción política bilateral entre China e Italia y no representaría la línea común del plan estratégico UE-China, sobre todo con referencia a las posibles repercusiones en las tradicionales alianzas de Europa con EEUU.

Sin embrago, entre tantas críticas al Gobierno italiano, la adhesión de Italia a la IFR a través de la firma del MoU constituye el compromiso del primer país miembro del G7 y país fundador de la UE al megaproyecto de cooperación de China, lo que lo caracteriza como país pionero en este asunto ${ }^{12}$. La firma del MoU que vincula Italia a China en la IFR representa un acto de amistad estratégica entre los dos países. De hecho, el Presidente Xi destacó "un pacto estratégico con Italia", definiendo esta visita como la evolución de una larga amistad entre los dos países símbolos de "la gran historia de los encuentros entre Oriente y Occidente, un nuevo capítulo para la amistad entre Roma y Pekín” (Xi, 2019). El Presidente Xi subrayó que las relaciones entre China e Italia celebrarán 50 años en el 2020, a pesar de que el conocimiento entre las culturas romanas y chinas empezó hace 2000 años y se ha renovado en muchas ocasiones durante la historia, como las misiones de Matteo Ricci y los viajes de Marco Polo en la antigua Ruta de la Seda: los primeros occidentales que

\footnotetext{
${ }^{9}$ Texto disponible en: https://eur-lex.europa.eu/eli/reg/2019/452/oj?locale=es

${ }^{10}$ Punto 6 de los Considerando del Reglamento 452/2019.

${ }^{11}$ Aparece en los documentos la controvertida denominación de China como un: "rival sistémico" y "competidor económico".

${ }^{12}$ No obstante, Italia no es el primer socio comercial de China. Está atrás de Alemania, Francia y Reino Unido.
} 
tuvieron un conocimiento profundo de la cultura china, y conocidos por ello como los "primeros sinólogos del mundo".

Analizando el MoU sobre IFR entre Italia y China observamos que sus efectos son esencialmente políticos y no inciden en las cuestiones jurídicas y económicas de la UE. De hecho, el MoU define con extrema claridad la colaboración de la IFR con los principios de la Agenda 2030 de la ONU, la Agenda 2020 de cooperación UE-China y la estrategia de la UE con la conectividad euroasiática de 2018. Por lo tanto, el MoU se confirma como herramienta totalmente en línea con el derecho internacional y los principios constitutivos de la ONU. Podemos destacar algunos puntos:

1. Las sinergias entre la IFR y las prioridades identificadas en el Plan de Inversiones para Europa y las Redes Transeuropeas de Transporte. La "Plataforma de Conectividad UE-China", destinada a mejorar la eficiencia; 2. El MoU retoma "la importancia de procedimientos de contratación abiertos, transparentes y no discriminatorios", basándose en el Reglamento UE 2019/452, que estableció un marco para el control de inversiones extranjeras directas en la UE; 3. Oposición al unilateralismo y al proteccionismo; 4. Mejora de la cooperación Norte-Sur, Sur-Sur y triangular; 5. Establecimiento del Diálogo Italia-China a nivel financiero entre el Ministerio de Economía y Finanzas de la República Italiana y el Ministerio de Finanzas de la República Popular China; 6. El Foro Cultural Italia-China para la ejecución de proyectos de hermanamiento entre sitios italianos y chinos registrados por la UNESCO como sitios del Patrimonio Mundial; 7. La implementación de la Agenda 2030 para el Desarrollo Sostenible y el Acuerdo de París sobre Cambio Climático. El Ministerio de Medio Ambiente y Protección del Territorio y el Mar de la República Italiana participará activamente en la Coalición Internacional para el Desarrollo Verde como parte de la IFR, lanzada por el Ministerio de Ecología y Medio Ambiente de la República Popular. China y el Programa de las Naciones Unidas para el Medio Ambiente (PNUMA); 8. Las Partes seguirán los principios del mercado, promoverán la colaboración entre el capital público y privado, fomentarán la inversión y el apoyo financiero a través de modelos diversificados; 9. El MoU no constituye un acuerdo internacional del cual puedan surgir derechos y obligaciones bajo el derecho internacional. Ninguna de las disposiciones de este Memorando debe interpretarse y aplicarse como una obligación o compromiso legal o financiero de las Partes. La interpretación de este Memorando de Entendimiento debe estar de acuerdo con las leyes nacionales de las Partes, así como con el derecho internacional aplicable y, en lo que respecta a la Parte italiana, con las obligaciones derivadas de la pertenencia de Italia a la UE (Artículo 11 de la Constitución italiana).

Como se puede observar, el MoU Italia-China no representa un acto "revolucionario", ya que se encuadra en el derecho internacional y en el derecho 
de la UE, incluido en el reglamento 452/2019. Sin embargo, este MoU es una fuerte señal política de cooperación integral con China, que se diferencia de las estrategias de Francia y de Alemania, que priorizaron el intercambio puramente económico.

La firma del MoU sobre la IFR marca una doble velocidad entre los países de la UE, entre los del Sur-Este y los del Norte. Hasta ahora los países de la UE que han ingresado oficialmente la IFR son 16. Los 13 Estados miembros que han firmado MoU específicos son: Bulgaria; Croacia; Republica Checa; Estonia; Hungría (el primero en firmar entre los estados de la UE, en 2015); Grecia; Letonia (el primero entre los países bálticos, en 2016); Lituania; Malta; Polonia; Portugal; Eslovaquia; y Eslovenia. A estos, se sumaron Italia y Luxemburgo (finales de marzo de 2019) y Chipre (septiembre de 2019). Ampliando el campo de investigación, resulta que otros países europeos, no miembros de la UE, han firmado acuerdos con China en el contexto de la IFR. Estos incluyen Turquía, Azerbaiyán, Bielorrusia, Rusia, Georgia, Ucrania y Moldavia.

Cabe destacar que la IFR ha puesto de relieve algunas zonas grises de Europa. Como primer resultado, la IFR ha generado entusiasmo en los Estados de la UE, aunque posteriormente la iniciativa ha sido duramente criticada por la falta de transparencia y una posible crisis del bloque debido a la práctica china de acuerdos bilaterales que habrían exacerbado el proceso de competencia entre los Estados miembros ${ }^{13}$. Estas comenzaron a extenderse debido a la presencia progresiva de China en los países de Europa del Este, lo que ha alimentado el temor europeo de una posible pérdida progresiva de influencia en estas áreas, y este punto nos remite a las cuestiones geopolíticas, que siempre están entrelazadas con la creciente interdependencia económica.

De hecho, el área de los Balcanes ha sido relegada por la UE como una "Europa de segundo nivel", que debería estar sujeta a su influencia política, legal y comercial, ya que estos países están a la espera de una posible membresía. En relación con la IFR, los Balcanes siempre han sido considerados como una "zona de tránsito" entre los dos grandes protagonistas: UE y China.

Considerando la posibilidad de ganar influencia en estos territorios, China y la UE se han embarcado en un camino antagónico, ambos se disputan la influencia en los Estados de esta región. De hecho, China ha iniciado una serie de reuniones llamadas " $16+1$ ", ahora " $17+1$ " (con el ingreso de Grecia): desde 2012 China ha involucrado a 16 países de Europa Central y Oriental, incluidos 12 Estados miembros de la UE (Bulgaria, Croacia, República Checa, Estonia, Hungría, Letonia, Lituania, Polonia, Rumania, Eslovaquia, Eslovenia y Grecia) y cinco países de los Balcanes Occidentales (Albania, Bosnia y Herzegovina, Montenegro, Serbia y Macedonia) como parte de un formato de cooperación

\footnotetext{
${ }^{13}$ Documento del Parlamento Europeo, China, the 16+1 cooperation format and the EU, 2017.
} 
alternativo, que representa un enfoque innovador de la cooperación regional (Jakóbowski, 2018). De hecho, según China, $17+1$ es un modo experimental para la cooperación regional que comparte características comunes con las otras plataformas de cooperación plurilateral que China ha creado con los países de África, Asia oriental, América Latina y el Medio Oriente.

Como era de esperar, la UE ha criticado enérgicamente este formato como un posible factor de desagregación para la integración europea (EuropeanParliament, 2017). La UE tiene un plan estratégico con China (EU-China, 2020) altamente desarrollado y considera a la plataforma $16+1$ como una desviación de la "regla", que no ha generado un verdadero multilateralismo, sino que ha aumentado el nivel de conflicto entre los países europeos. Sin embargo, en 2018, la actitud europea cambió para mejor, como se indica en el documento del Parlamento Europeo (European-Parliament, 2018), especialmente desde que la UE es observadora del $16+1$ y con el desarrollo progresivo de la iniciativa europea del "Berlin Process 2014-2018"14.

Para afirmar aún más su apoyo a la integración europea, en la Agenda Estratégica para la cooperación UE-China 2020 y en las Directrices para la cooperación entre China y los países de Europa del Este adoptados en la última reunión $16+1$ en Sofía, China ha declarado su apoyo a la integración europea y ha considerado el formato de cooperación $16+1$ como "un complemento en lugar de una alternativa a la asociación estratégica global UE-China" (SCPRCCHINA, 2018). "La cooperación $16+1$ no es en absoluto una plataforma geopolítica. Algunos pueden decir que tal cooperación puede separarse de la UE, pero eso no es verdad", ha declarado el primer ministro chino, Li Keqiang.

Esta posición china depende de varios factores: China no puede dejar de lado sus compromisos con la UE en el marco de la IFR así como en la dinámica de la cooperación estratégica integral que tiene con la UE. Por otro lado, China ha destacado que los países balcánicos, a pesar de no ser todavía miembros de la UE, de hecho actúan en el marco legal de la Unión Europea. El proceso de Berlín ofrece una plataforma de cooperación orientada a resultados entre la UE, los Balcanes Occidentales y los socios chinos.

Lo que está ocurriendo en Europa es un doble proceso propulsivo de integración regional: por un lado, se está buscando una visión común hacia la IFR en el marco de los países de la UE en un momento histórico de gran fragmentación y euroescepticismo; por otro lado, la IFR y Berlin Process están

${ }^{14}$ El Berlin-Process comenzó en 2014 como una iniciativa de la canciller alemana, Angela Merkel y se ha convertido en una plataforma útil para mejorar la cooperación regional, para apoyar proyectos de conectividad y para presionar a los Estados del sudeste de la UE a realizar reformas estructurales y la adhesión a la UE. Como lo mencionó el Presidente de la Comisión Europea en su "Estado de la Unión", discurso pronunciado en septiembre de 2017, la perspectiva de la ampliación es creíble y realista. Los estados miembros de la UE han demostrado su compromiso facilitando el proceso de transformación de los Balcanes Occidentales con la cooperación a nivel regional. Esto implica esfuerzos comunes que podrían y pueden lograr resultados tangibles mediante el Berlin-Process. 
sobrellevando una dinámica integrativa e inclusiva de los países balcánicos (BERLIN-PROCESS, 2018), que hasta ahora habían quedado afuera de la visión europea. Hoy son vistos como potenciales miembros (Marciacq, 2018).

Asimismo, la pandemia COVID-19 ha funcionado como elemento detonador y acelerador de esta tendencia. El COVID-19 funcionó como un suero de la verdad, haciendo que el velo de Maya cayera en la narrativa mística de Europa. En el comienzo de la Pandemia se observó un abandono de Italia por parte de sus socios europeos (Staiano, 2020).

Un asunto que poco se ha discutido fue la espera predeterminada de Alemania y Francia en hacer públicos los contagios en sus respectivos territorios a expensas del colapso económico de Italia y la humillación que sufrió al verse obligada a pedir la no aplicación del fiscal compact ${ }^{15}$.

Lo más grave es que Alemania ha pasado por alto las instituciones europeas al poner en práctica medidas económicas nacionales, que no aparecen en el presupuesto nacional. De hecho, Alemania ha asignado $550 \mathrm{mil}$ millones de euros (en comparación con los 25 italianos) puestos a disposición del Kreditanstalt für Wiederaufbau (KFW), con "asistencia ilimitada" a las empresas alemanas, así como ha declarado el ministro de Finanzas alemán, Olaf Scholz ${ }^{16}$.

Profundos desacuerdos preexistentes entre los miembros de la UE estallaron durante la emergencia de la pandemia del COVID-19, y generaron fracturas en el proceso de integración europea. Y el caso italiano se tornó paradigmático. El Primer Ministro italiano Conte, durante la reunión del CE del 26 de marzo de 2020, reaccionó con dureza a la oposición alemana exigiendo ayuda para los países europeos más afectados a través del Banco Central Europeo. Conte pidió claramente la adopción de "herramientas innovadoras" y no el uso de "viejas fórmulas personalizadas"17. El presidente de la República Italiana, Sergio Mattarella, reiteró además la necesidad de un enfoque nuevo y no limitado a los “esquemas predeterminados" (referido al enfoque alemán) no aplicable en esta emergencia, y enfatizó que "la solidaridad no es solo un requisito de los valores de la UE; también es de nuestro interés común”18, haciendo hincapié

${ }^{15}$ El fiscal compact es la expresión con la cual es conocido el Tratado de estabilidad, coordinación y gobernanza en la unión económica y monetaria, un tratado internacional aprobado el 2 de marzo de 2012 por 25 de los 28 estados miembros de la Unión Europea (no fue firmado por el Reino Unido, Croacia y la República Checa). Entró en vigor el 1 de enero de 2013, el pacto contiene el principio vinculante del equilibrio presupuestario.

${ }^{16}$ El KFW no es un banco sino un organismo público, un accionista del Kfw Ipex-Bank, que realiza actividades bancarias, pero no supera el umbral de 30 mil millones: por esta razón está exento de la supervisión del BCE, no debe cumplir con los requisitos de capital y reglas de la unión bancaria. Prácticamente opera como un banco central que responde al gobierno, que es su accionista de referencia.

${ }^{17} \mathrm{https} / /$ www.repubblica.it/economia/2020/03/26/news/conte_alla_ue_se_aiuti_gli_saranno_come_ in_passato_facciamo_da_soli_-252410637/

${ }^{18} \mathrm{https}$ ://www.quirinale.it/elementi/48604 
en una posible estrategia de salida italiana de la UE (Italexit). El apoyo de los primeros ministros de Francia, España y Portugal indica, como expuesto anteriormente, una profunda brecha entre el sur de Europa y el norte de Europa. Las declaraciones de la presidenta del BCE, Cristine Lagarde, contra Italia, así como la de la presidenta de la CE, Ursula von der Leyen, que se disculpó formalmente con Italia tras haber subestimado el impacto de la pandemia, son una clara señal de las relaciones complejas e inestables entre las dos partes de Europa.

En marzo de 2020, en el peor momento del impacto del COVID-19 en Italia, el sentimiento de los italianos era resumido con la declaración del filósofo italiano Massimo Cacciari: "sólo China salvará Italia” (Bedini Crescimanni, 2020).

\section{Conclusiones}

Por más de tres décadas la globalización neoliberal ha convivido con el proceso de expansión y consolidación de regionalismo europeo por intermedio de la experiencia supranacional, la UE, y con un alineamiento geopolítico con los EEUU que viene desde el origen de la Guerra Fría y que tiene a la OTAN como eje. Los últimos años estamos presenciando un fenómeno transformativo interesante en estos dos planos.

En el plano geopolítico, inherentemente ligado a la lógica territorial de los Estados (Arrighi, 1996), la pandemia del COVID-19 reforzó la rivalidad entre EEUU y China que tiene su correlato en la guerra comercial y tecnológica. También quedaron más evidentes las disputas de la UE con China. La dinámica de las relaciones económicas está ligada a la geopolítica. El ascenso de China y su fortalecimiento económico derivaron en un importante crecimiento del comercio Europa-China y de las inversiones de China en Europa ${ }^{19}$. La creación de la IFR no es sino el resultado más acabado de este proceso. Ella abre un espacio de acción y diversificación de opciones para la UE, pero al mismo tiempo crea un dilema geopolítico para el bloque con el que tendrá que convivir.

En primer lugar, la IFR expone las desigualdades regionales en la región europea y las limitaciones de la globalización neoliberal que profundizan las desigualdades en el interior de los países y que estimulan el crecimiento del euroescepticismo y de los diferentes populismos de derecha. La Pandemia de COVID-19 aceleró este proceso y modificó las expectativas con la cooperación en ayuda que China realizó a varios países de Europa en el peor momento de la pandemia.

${ }_{19} \mathrm{Si}$ bien hubo recentemente uma disminución de las inversiones chinas em Europa em general, eso fue uma tendência em todas las regiones (Kratz, Huotari, Hanemann, \& Arcesati, 2020) 
El caso italiano hace converger estos dilemas de una manera dramática, ya que se trata de un miembro del G7 y de unos de los actores más importantes de la UE. Italia es el punto focal de la discusión en torno a la IFR, de las tensiones Norte-Sur dentro de la UE y de las relaciones socioculturales entre Occidente y China.

En 2020, las placas tectónicas geopolíticas y económicas continuarán en movimiento a ritmos asincrónicos en un año pandémico. El COVID-19 actuó como game-changer que estimuló los cambios adaptativos en la UE. No solo las percepciones en relación con China, sino con los EEUU también. La Comisión europea se autodenominó, antes de la pandemia, una "Comisión geopolítica" (European Comission, 2019) y recientemente el Alto Representante de la UE, Josep Borrell, anunció la "doctrina Sinatra" que si bien no defiende una equidistancia ${ }^{20}$ entre EEUU y China, reivindica una autonomía frente al nuevo escenario internacional (Borrell, 2020) y una mayor cooperación entre los miembros de la UE.

La cooperación proactiva de China en la Pandemia por intermedio de la "diplomacia de las masacrillas" y las críticas que sufrieran los países ricos de la UE, especialmente Alemania, por parte de los países del Sur europeo (Italia, España, Grecia y Portugal) provocó una respuesta específica por parte de la UE: el Paquete de Recuperación del Coronavirus ${ }^{21}$ (DW, 2020). Los líderes de la UE llegaron a un acuerdo de ayuda sin precedentes de U\$S 2 billones y un acuerdo presupuestario destinado a ayudar a los miembros del bloque más afectados. No hay consenso en torno al impacto de esta medida. Algunos economistas presentan un panorama más optimista, ya que por primera vez los Estados miembros podrán endeudarse en condiciones de largo plazo y con grandes facilidades (Mazzucato, 2020); otros, como Varoufakis, prevén un crecimiento de desigualdad dentro de los Estados miembros, lo que desencadenaría más euroescepticismo y fragmentación social en el futuro (Varoufakis, 2020).

La UE deberá lidiar con dos modelos de globalización en un ambiente geopolítico particular de gran rivalidad China-EEUU. La aceptación de la IFR en las normas de la UE implica aceptar el liberalismo integrado, pero con características chinas, con la carga cultural que eso implica. Por otro lado, la reacción del paquete de ayuda permanece en la incógnita. ¿Transitará hacia un nuevo pacto social inclusivo que fortalecerá la UE superando las soluciones clásicas de austeridad o se trata del mismo remedio neoliberal con otro rótulo, que fragmentará aún más el tejido social y acelerará la polarización entre sus miembros ricos y pobres?

\footnotetext{
${ }^{20}$ Borrell reconoce mayores proximidadades culturales e históricas com EEUU.

${ }^{21}$ El paquete incluye un fondo de $€ 750$ mil millones que pueden estar disponibles como créditos y subsidios, así como un presupuesto de 1 billón de euros por siete años para la UE.
} 


\section{Referencias bibliográficas:}

Amin, Samir. (2006). Os desafios da Mundialização. Aparecida (SP): Idéias \& Letras.

Arrighi, Giovanni. (1996). O Longo Século XX. São Paulo: Contraponto.

Arrighi, Giovanni. (2007). Adam Smith in Beijing: lineages of the twenty-first century. London; New York: Verso.

Arrighi, Giovanni, Silver, Beverly J., \& Ahmad, Iftikhar. (1999). Chaos and governance in the modern world system. Minneapolis: University of Minnesota Press.

Bedini Crescimanni, Tommaso (2020). Massimo Cacciari: 'Il coronavirus è la pietra tombale sull'integrazione europea, sarà la Cina a risollevare l'Italia'. Business Insider Italy, Marzo 16. https://it.businessinsider.com/ massimo-cacciari-il-coronavirus-e-la-pietra-tombale-sullintegrazioneeuropea-sara-la-cina-a-risollevare-litalia/

BERLIN-PROCESS. (2018). The Berlin Process 2014-2018 Report Berlin Process Series, February. https://wbc-rti.info/object/document/17032

Borrell, Joseph. (2020). La Doctrina Sinatra. Política Exterior, Agosto 27(197). https://www.politicaexterior.com/producto/la-doctrina-sinatra/

Cahill, Damien. (2014). The end of laissez-faire?: on the durability of embedded neoliberalism. Cheltenham UK - Northampton USA: Edward Elgar Pub.

Cairo Carou, Heriberto. (2018). Las guerra 'virtuosas' de George W. Bush. Madrid: Trama Editoriales.

China-SCPRC. (2011). China's Peaceful Development. September. http://english.www.gov.cn/archive/white_paper/2014/09/09/ content $281474986284646 . \mathrm{htm}$

DW. (2020). EU leaders reach deal on coronavirus recovery package. $D W$, July 21. https://www.dw.com/en/eu-leaders-reach-deal-on-coronavirusrecovery-package/a-54242834

EC-China. (2017). Memorandum of Understanding on a dialogue in the area of the State aid control regime and the Fair Competition Review System. June 2. https://ec.europa.eu/competition/international/bilateral/mou china 2017.pdf

EU-China. (2020). EU-China Summit: Defending EU interests and values in a complex and vital partnership. June 22. https://www.consilium.europa. $\mathrm{eu} / \mathrm{it} / \mathrm{press} / \mathrm{press}-\mathrm{releases} / 2020 / 06 / 22 / \mathrm{eu}$-china-summit-defending-euinterests-and-values-in-a-complex-and-vital-partnership/

European Comission, EC (2019). The von der Leyen Commission: for a Union that strives for more. European Comission, September 10. https:// ec.europa.eu/commission/presscorner/detail/en/IP $19 \quad 5542$ 
European-Parliament. (2017). China, the $16+1$ format and the EU. Parliament Policy Brief. $\quad$ https://www.europarl.europa.eu/RegData/etudes/ BRIE/2018/625173/EPRS BRI(2018)625173 EN.pdf

European-Parliament. (2018). China, the $16+1$ format and the EU. European Parliament Policy Brief. https://www.europarl.europa.eu/RegData/etudes/ BRIE/2018/625173/EPRS BRI(2018)625173 EN.pdf

Gill, Chris (2020). CCP announces plan to take control of China's private sector. Asia Times Financial, Sept 17. CCP announces plan to take control of China's private sector

Harvey, David. (2005). A Brief History of Neoliberalism. New York: Oxford University Press.

Held, David, \& McGrew, Anthony. (2000). The Global Transformations Reader: An Introduction to the Globalization Debate. Cambridge/Malden: Polity Press.

Hu, Jintao. (2012). Texto íntegro del informe presentado por Hu Jintao en XVIII Congreso del PCCh. November 11. http://cr.chineseembassy.org/ esp/zt/t992906.htm

Kratz, Agatha, Huotari, Mikko, Hanemann, Thilo, \& Arcesati, Rebecca. (2020). Chinese FDI in Europe: 2019 update April https://www.merics. org/sites/default/files/2020-04/MERICS-Rhodium\%20Group_COFDIUpdate-2020_2.pdf

Li, Xing. (2015). Interpreting and Understanding "The Chinese Dream" in a Holistic Nexus. Fudan J. Hum. Soc. Sci., 8(4), 505-520. doi:10.1007/ s40647-015-0098-3

Liu, Weidong, \& Dunford, Michael. (2016). Inclusive globalization: unpacking China's Belt and Road Initiative. Area Development and Policy, 1(3), 323340. doi:10.1080/23792949.2016.1232598

Marciacq, Florent. (2018). The European Union and the Western Balkans after the Berlin Process Reflecting on the EU Enlargement in Times of Uncertainty. Dialogue SOE. https://library.fes.de/pdf-files/bueros/ sarajevo/13948.pdf

Mazzucato, Mariana. (2020). The success of the EU recovery fund will depend on bold missions. Financial Times, August 9. https://www.ft.com/content/ b26f6785-e08a-450b-8c62-bdafbeb5ff2c

Morin, Edgar. (2001). Introducción al pensamiento Complejo. http:// cursoenlineasincostoedgarmorin.org/images/descargables/Morin Introduccion_al_pensamiento_complejo.pdf 
National Development and Reform Commission. (2015). Vision and Actions on Jointly Building Silk Road Economic Belt and 21st-Century Maritime Silk Road. National Development and Reform Commission, Ministry of Foreign Affairs, and Ministry of Commerce of the People's Republic of China, March 28. http://en.ndrc.gov.cn/newsrelease/201503/ t20150330 669367.html

Oke, Nicole. (2009). Globalizing Time and Space: Temporal and Spatial Considerations in Discourses of Globalization. International POlitical Sociology, 3, 310-326.

Polanyi, Karl. (2000). A Grande Transformação: as origens de nossa época (2a ed.). Rio de Janeiro: Campus.

Qin, Yaqing. (2014). Continuity through Change: Background Knowledge and China's International Strategy. The Chinese Journal of International Politics, 7(3), 285-314. doi:10.1093/cjip/pou034

Scholte, Jan Aart. (2005). The Sources of Neoliberal Globalization. United Nations Research Institute for Social Development. http://www.unrisd. org/80256B3C005BCCF9/search/9E1C54CEEB19A314C12570B4004D 0881? OpenDocument

SCPRC-CHINA. (2018). The Sofia Guidelines for Cooperation between China and Central and Eastern European Countries. Xinhua, July 16. http://english.www.gov.cn/news/international_exchanges/2018/07/16/ content_281476224693086.htm

Sheppard, Eric S. (2016). Limits to globalization : disruptive geographies of capitalist development (First edition. ed.). Oxford: Oxford University Press.

Staiano, Maria Francesca. (2018a). La relaciones internacionales entre China y América Latina: encontrando un camino común hacia un nuevo orden mundial. Humania del Sur. Revista de Estudios Latinoamericanos, Africanos y Asiáticos, Julio-Diciembre(25).

Staiano, Maria Francesca. (2018b). Xi Jinping: el hombre fuerte designado por el Partido. Opiniones en el IRI. http://www.iri.edu.ar/wp-content/ uploads/2018/03/opiniones-en-el-iri-staiano-marzo.pdf

Staiano, Maria Francesca. (2020). "Para Europa doblan las Campanas". Opiniones en el IRI. http://www.iri.edu.ar/index.php/2020/03/09/paraeuropa-doblan-las-campanas/

Staiano, Maria Francesca , \& Bogado Bordazar, Laura Lucía (2019). La iniciativa la Franja y la Ruta: innovación propulsora de los procesos de integración regional a nivel global. Los casos de Europa y América Latina. In Laura Lucía Bogado Bordazar, Maria Francesca Staiano, \& Matías Caubet (Eds.), China: una nueva estrategia geopolítica global: la iniciativa la Franja y la Ruta La Plata: Universidad Nacional de La Plata. 
The Lancet. (2017). Facing forwards along the Health Silk Road. The Lancet - Global Health, 5(10). doi:https://doi.org/10.1016/S2214109X(17)30349-2

Vadell, Javier. (2018). El Foro China-CELAC y el nuevo regionalismo para un mundo multipolar: desafíos para la Cooperación 'Sur-Sur'. Carta Internacional, 13(1), 6-37. doi:https://doi.org/10.21530/ci.v13n1.2018.733 Varoufakis, Yanis. (2020). The EU coronavirus fund will take Europe another step towards disintegration. The Guardian, July 24. https://www. theguardian.com/world/commentisfree/2020/jul/24/eu-coronavirus-fundeurope-recovery-package

Vines, David (2017). Opinion: One Belt, One Road: China's 21st Century Marshall Plan? Caixin, May 17. https://www.caixinglobal.com/2017-05-17/ opinion-one-belt-one-road-chinas-21st-century-marshall-plan-101091483. $\underline{\mathrm{html}}$

Xi, Jinping. (2015). Working Together to Forge a New Partnership of Win-win Cooperation and

Create a Community of Shared Future for Mankind. UN https://gadebate.un.org/ sites/default/files/gastatements/70/70 ZH_en.pdf

Xi, Jinping. (2017a). Texto íntegro del informe presentado por Xi Jinping ante XIX Congreso Nacional del PCCh. http://spanish.xinhuanet.com/201711/03/c 136726335.htm

Xi, Jinping. (2017b). Work Together to Build a Community of Shared Future for Mankind. Xinhuanet, January 18. http://www.xinhuanet.com/english/2017$\underline{01 / 19 / \mathrm{c} 135994707 . \mathrm{htm}}$

Xi, Jinping. (2019, Marzo 20). La visita di Xi Jinping: «Un patto strategico con l'Italia». Corriere della Sera. Retrieved from https://www.corriere.it/ esteri/19 marzo 20/patto-strategicoassieme-all-italia-efa36c0c-4a8c-11e9a7a3-5683e4dbacbc.shtml

Yan, Xuetong. (2014). From Keeping a Low Profile to Striving for Achievement. The Chinese Journal of International Politics, 7(2), 153-184. 
